\title{
Molecular dynamics simulations of nanometric metallic multilayers: Reactivity of the Ni-Al system
}

\author{
Florence Baras and Olivier Politano \\ Laboratoire Interdisciplinaire Carnot de Bourgogne, UMR 5209 CNRS-Université de Bourgogne, 9 Avenue A. Savary, \\ Boite Postale 47 870, F-21078 Dijon Cedex, France
}

(Received 17 May 2011; published 12 July 2011)

\begin{abstract}
The reactivity of a layered $\mathrm{Ni}-\mathrm{Al}-\mathrm{Ni}$ system is studied by means of molecular dynamics simulations, using an embedded-atom method type potential. The system, made of an fcc-Al layer embedded in fcc-Ni, is initially thermalized at the fixed temperature of $600 \mathrm{~K}$. The early interdiffusion of $\mathrm{Ni}$ and $\mathrm{Al}$ at interfaces is followed by the massive diffusion of $\mathrm{Ni}$ in the $\mathrm{Al}$ layer and by the spontaneous phase formation of B2-NiAl. The solid-state reaction is associated with a rapid system heating, which further enhances the diffusion processes. For longer times, the system may partly lose some its $B 2-\mathrm{NiAl}$ microstructure in favor of the formation of $L 1_{2}-\mathrm{Ni}_{3} \mathrm{Al}$. This stage is controlled by the diffusion of $\mathrm{Al}$ in the Ni-rich phase, and a layer-by-layer development of the new phase is observed.
\end{abstract}

DOI: 10.1103/PhysRevB.84.024113

PACS number(s): 64.70.Nd, 02.70.Ns, 68.35.bd

\section{INTRODUCTION}

Nanometric metallic multilayers (N2M's) are obtained by the superposition of very thin $A-B$ films, where $A$ and $B$ are pure metals. The layer thickness typically varies between a few to 100 nanometers and the number of layers may be extremely large, about 5000. Such multilayer nanofilms are now easily produced by a magnetron sputtering technique or high-vacuum deposition (for a relatively small number of layers). Because of their interesting applications in many modern technologies, ${ }^{1-3}$ the production of N2M's has increased considerably during the past decade. Concomitantly, their study attracts more and more attention (for a recent review, see Ref. 4).

The N2M is chemically stable if $A$ and $B$ are nonmiscible. Otherwise, $A$ and $B$ will first mix into a solid solution and then react to give intermetallics compounds. Since the material is made of alternating very thin layers, it may become highly reactive even at low temperature and the reaction proceeds quite rapidly. N2M's are thus well suited for welding and soldering heat-sensitive materials in a few seconds without damage. The performance and the efficiency of N2M are directly related to the promotion or the inhibition of reactions such as compound formation, crystallization, or grain growth.

To understand the behavior of such systems, it is thus necessary to study the dynamics of phase and structural transformations associated with heterogeneous reactions at nanoscale. Since this kind of nano-object is essentially made of interfaces, it raises many questions about the role of huge concentration gradients in the nucleation process, the limiting role of diffusion on the reaction, the presence of defects due to the misfit in the lattice parameters, and the real possibility of crystallization in nanosystems. Besides the very powerful experimental techniques that are now available to study N2M's, such as time-resolved x-ray diffraction (TRXRD) using synchrotron radiation or differential scanning calorimetry (DSC), it is interesting to develop an appropriate modeling of these nanosystems. Molecular dynamics (MD) seems to be a promising tool to study N2M's since the typical length scales (a few nanometers) correspond precisely to the ones accessible by the simulation. Moreover, a great effort has been made to develop interatomic potentials for binary metallic systems and their alloys.
Molecular dynamics has been used successfully to study the interface between crystalline copper and liquid aluminum, ${ }^{5}$ the demixing phenomena in NiAl nanosized particles, ${ }^{6}$ the melting and crystal growth in $\mathrm{Al}_{50} \mathrm{Ni}_{50}$ system, ${ }^{7}$ the sintering process and the alloying between $\mathrm{Al}$ and Ni nanoparticles, ${ }^{8}$ the exothermic alloying of $\mathrm{Ni} / \mathrm{Al}$ multilayers induced by shock loading,,${ }^{9,10}$ or to determine the pressure-dependent melting temperature of $\mathrm{Al}$ and $\mathrm{Ni}^{11}{ }^{11}$ This method has also been used to investigate the interfacial mixing behavior in transition metal $(\mathrm{Fe}, \mathrm{Co}, \mathrm{Ni})-\mathrm{Al}$ multilayer systems during deposition ${ }^{12}$ or to study the atomic scale structure of sputtered metal multilayers. ${ }^{13}$

\section{DETAILS OF THE SIMULATION}

In this work, we study a layered Ni-Al-Ni system by molecular dynamics using an embedded-atom method (EAM). ${ }^{14} \mathrm{We}$ use the specific potential developed by Mishin et al. ${ }^{15}$ that has been constructed for the intermetallic compound $B 2-\mathrm{NiAl}$ as well as for $\mathrm{Ni}$ and $\mathrm{Al}$ by fitting to both experimental properties and $a b$ initio data. The potential accurately reproduces the basic lattice properties of $\mathrm{B} 2-\mathrm{NiAl}$ and the energetics and stability of other structures like $L 1_{2}-\mathrm{Ni}_{3} \mathrm{Al}$. As compared to a real Ni-Al system, the potential deals with a simplified system with a limited number of phases. The MD simulation is conducted using the LAMMPS software package. ${ }^{16}$

We choose a simplified geometry (see Fig. 1) of a layered $\mathrm{Ni}-\mathrm{Al}-\mathrm{Ni}$ system to model alternating continuous very thin layers of $\mathrm{Ni}$ and $\mathrm{Al}$. The system is made of one layer of $\mathrm{Al}$ (9 atomic planes form the inner layer) in between two $\mathrm{Ni}$ layers of 11 atomic planes each (the outer layers) along the $z$ direction. Both pure metals are in fcc configuration and the interface orientation is (001). The width in the $x$ and $y$ directions of the $\mathrm{Al}$ layer is chosen to be smaller than the Ni layers $\left[L_{x}(\mathrm{Al})<L_{x}(\mathrm{Ni})\right.$ and $\left.L_{y}(\mathrm{Al})<L_{y}(\mathrm{Ni})\right]$. The typical size of the simulation box is $L_{x}=L_{y} \approx 10 \mathrm{~nm}$ and $L_{z}=5.6 \mathrm{~nm}$. The system is submitted to periodic boundaries conditions in all directions. The specific geometry with a narrower Al layer allows us to avoid artificial constraints due to the application of periodicity on both $\mathrm{Al}$ and $\mathrm{Ni}$ layers during the construction of the system. This way, the system around the interface is able to manage the misfit between the two 


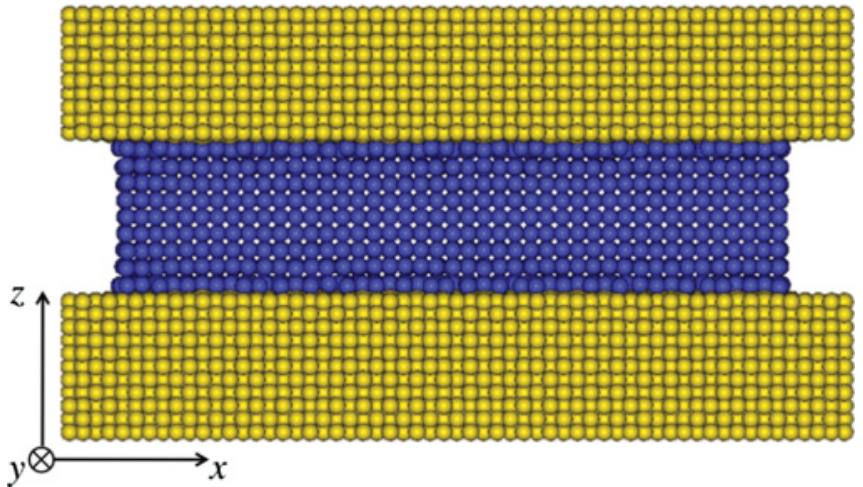

FIG. 1. (Color online) Initial configuration of the simulated system with one $\mathrm{Al}$ slice in between two Ni layers. $\mathrm{Al}$ and $\mathrm{Ni}$ are shown as blue (black) and yellow (white) spheres, respectively.

crystalline structures. It also appears that the initial distance $\delta$ between the Ni- and $\mathrm{Al}$-atomic planes around the interface may considerably influence the later evolution. A detailed analysis will be presented in a forthcoming publication. In this work, we present the results obtained for a value of $\delta=2.5 \AA$, which corresponds to a system's potential energy close to its minimum at $0 \mathrm{~K}$ and allows us to preserve the fcc local structure of the majority of both $\mathrm{Ni}$ and $\mathrm{Al}$ atoms. In this case, the $\mathrm{Al}$ layer is in traction [the interlayer distance $d_{i j}$ slightly exceeds the lattice parameter $\left.a_{0}(\mathrm{Al})\right]$ while the Ni layer is somewhat shrunk $\left[d_{i j}<a_{0}(\mathrm{Ni})\right]$.

The system is made of 43643 atoms $[n(\mathrm{Al})=8321$ and $n(\mathrm{Ni})=35322]$. Both $\mathrm{Al}$ and $\mathrm{Ni}$ lattice parameters were determined at $600 \mathrm{~K}$ on bulk systems by using isothermalisobaric (NPT) simulations (see Table I). Hence, the Ni-Al$\mathrm{Ni}$ system is created at $600 \mathrm{~K}$ with the appropriate lattice parameters and thermalized using simulations in the canonical statistical ensemble $(N V T)$ over 400 ps. The simulation is then carried out in the microcanonical statistical ensemble $(N V E)$ over more than $20 \mathrm{~ns}$. A snapshot of the system at $t=6 \mathrm{~ns}$ is shown in Fig. 2.

Several indicators allow us to follow the evolution of the system at the microscopic level. The number density profiles in the direction perpendicular to the interface give an indication of the crystallinity state and of the local composition $n_{i}$ in Ni or $\mathrm{Al}$ in each slice $i$. Well-defined peaks are typically associated with a solid system structured in atomic planes. If we calculate the local lattice structure according to the formulation given by Ackland, ${ }^{17}$ we get the local configuration around a given atom based not on the distance between particles but the angles. It is

TABLE I. Lattice parameter $a_{0}(\AA)$ and cohesive energy $E_{0}(\mathrm{eV})$ of the $\mathrm{Ni}-\mathrm{Al}$ compounds at $600 \mathrm{~K}$. The second column corresponds to the lattice structure. The notation $E_{\mathrm{Ni}}$ indicates the potential energy of an atom of $\mathrm{Ni}$ in a given structure. The last column is the atomic density $\rho\left(\right.$ at $\left./ \mathrm{nm}^{3}\right)$ at $600 \mathrm{~K}$.

\begin{tabular}{lcccccc}
\hline \hline Phase & & $a_{0}$ & $E_{0}$ & $E_{\mathrm{Al}}$ & $E_{\mathrm{Ni}}$ & $\rho$ \\
\hline $\mathrm{Ni}$ & $A 1$ & 3.482 & -4.493 & & -4.493 & 94.75 \\
$\mathrm{Ni}{ }_{3} \mathrm{Al}$ & $L 1_{2}$ & 3.561 & -4.593 & -3.295 & -5.025 & 88.58 \\
$\mathrm{NiAl}$ & $B 2$ & 2.886 & -4.460 & -3.551 & -5.369 & 84.37 \\
$\mathrm{Al}$ & $A 1$ & 4.087 & -3.359 & -3.359 & & 58.59 \\
\hline \hline
\end{tabular}

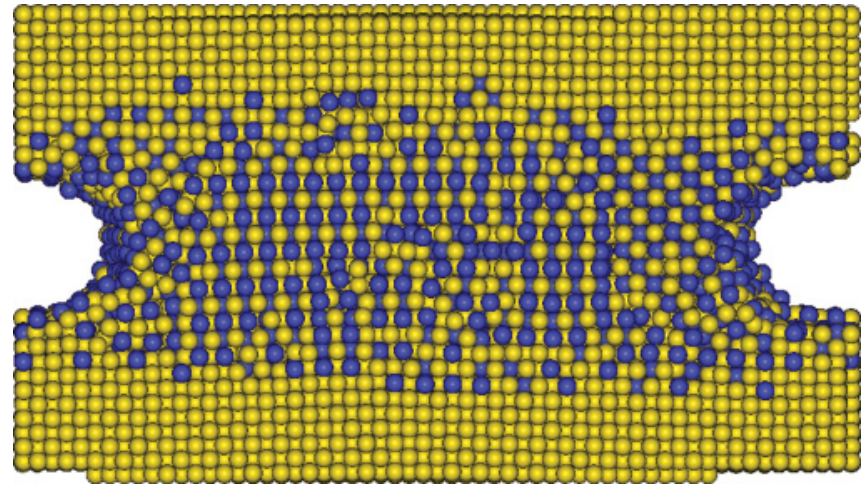

FIG. 2. (Color online) Snapshot of the simulated configuration with two Ni-Al interfaces, at $t=6 \mathrm{~ns}$, sliced for $y>10 \AA$. Al and $\mathrm{Ni}$ are shown as blue (black) and yellow (white) spheres, respectively. We note that the middle $\mathrm{Al}$ layer is curved due to the wetting.

possible to distinguish between five configurations: bcc ( $a=$ $1)$, fcc $(a=2)$, hcp, ico, and unknown. So each atom is labeled by the color of the species and by its local lattice structure. To determine the phase to which a given atom belongs, its potential energy can be compared to the potential energy of the same species atom in the structure corresponding to the phase. As shown in Table I, the potential energy of $\mathrm{Ni}$ in pure $\mathrm{Ni}$, in $\mathrm{NiAl}$, or in $\mathrm{Ni}_{3} \mathrm{Al}$ may be significantly different. To identify the lattice arrangement of a set of atoms (for instance, in an atomic plane), it is also possible to compute the histogram of pairwise distances (hpd) for all atoms in the set. This gives a quantity very similar to an unnormalized radial distribution function. This quantity can then be compared to the successive interatomic distances of a given structure, such as, for instance, B2-NiAl.

\section{RESULTS}

During the thermalization period at $600 \mathrm{~K}$, the main transformations appear in the inner layer. To deal with the crystallographic misfit and with the traction, $\mathrm{Al}$ atoms wet the free surface of $\mathrm{Ni}$ at the interfaces. This leads to the formation of defects in the $\mathrm{Al}$ atomic planes as vacancies or line defects that break the perfect symmetry of the planes. Sectors of fcc $\mathrm{Al}$ atoms are separated by two-dimensional grain boundaries. Due to the spontaneous adjustment of $\mathrm{Al}$ atoms close to the interface, the interplane distance $d_{i j}$ in the inner $\mathrm{Al}$ layer becomes very close to the lattice parameter of $\mathrm{Al}$ at $600 \mathrm{~K}$.

During the $N V E$ simulation, it is interesting to follow global quantities for the overall system. Figure 3 shows the evolution of the temperature together with the total number of atoms that are in a local configuration bcc and fcc. The temperature rise is accompanied with a structural modification of the system as fcc atoms are disappearing in favor of bcc atoms. In other words, while the total energy is conserved, there is an exchange between potential and kinetic energy in the system. It is particularly interesting to understand the spontaneous evolution of the system, in particular the development of an exothermic behavior, which reveals the extreme reactivity of the system. For this purpose, we analyze the different stages in the evolution. 


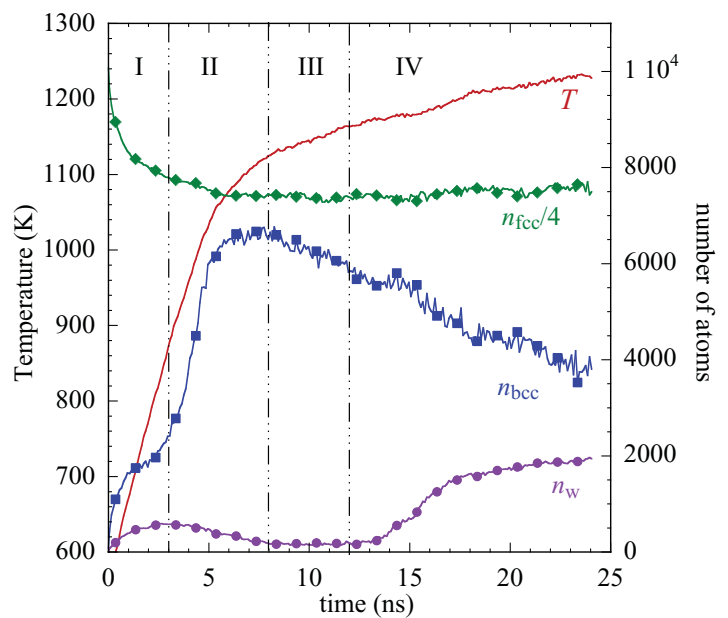

FIG. 3. (Color online) Temperature $T$ (scale in $\mathrm{K}$, on the left) and number of atoms (on the right) as a function of time (scale in $\mathrm{ns}$ ). The number of atoms in local configuration bcc $(a=1)$ is indicated by squares, one-quarter of the number of atoms in local configuration fcc $(a=2)$ by diamonds, and the number of wetting atoms $n_{w}$ by bullets. The different stages in the evolution are indicated by the dashed-dotted lines.

Just after the thermalization, from $t=0.4$ to $3 \mathrm{~ns}$ (stage I), the Al slice becomes amorphous. There is no more clear evidence of atomic planes and $\mathrm{Ni}$ atoms start to invade the zone, as shown in Fig. 4(a). On the other hand, the $\mathrm{Al}$ atoms diffusing in the $\mathrm{Ni}$ slices occupy the empty places liberated by outgoing $\mathrm{Ni}$ atoms and the atomic planes are not altered by the presence of $\mathrm{Al}$ atoms. As soon as the quantity of $\mathrm{Ni}$ is sufficient, the middle slice recovers its crystallinity. During

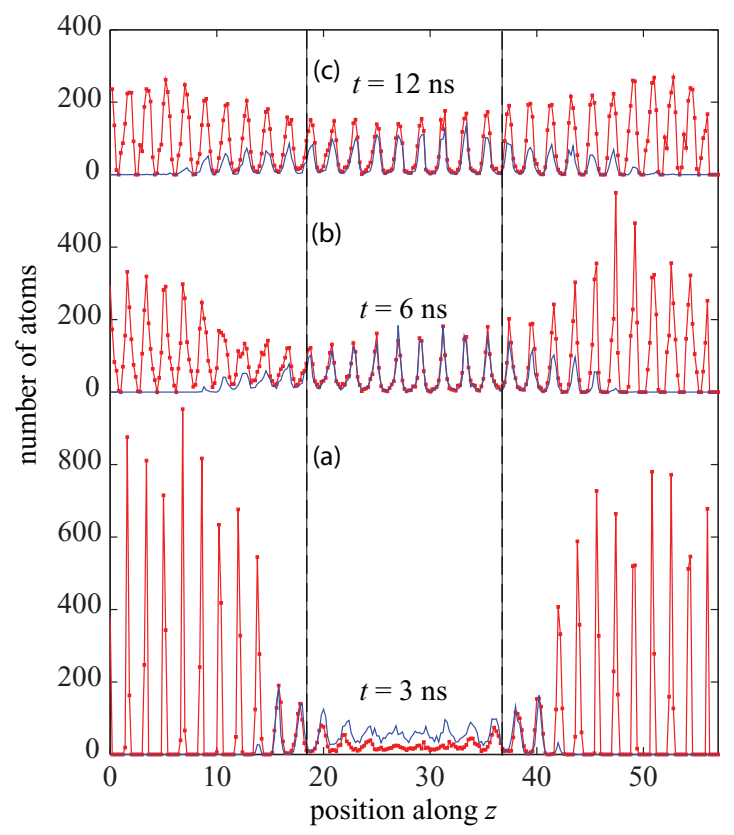

FIG. 4. (Color online) Number density profiles at $t=3,6$, and $12 \mathrm{~ns}$ in the direction $z$, perpendicular to the interfaces. The dashed lines indicate the limits of the inner Al layer in the initial configuration. The Ni profile is plotted with small bullets and the full line corresponds to the $\mathrm{Al}$ profile. Position is measured in angstroms.

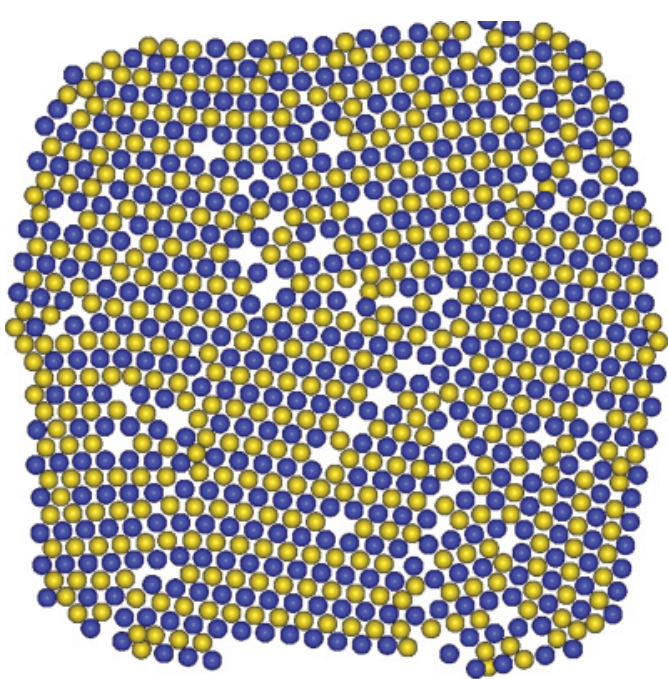

FIG. 5. (Color online) Snapshot of a slice $(24 \AA<z<26 \AA)$ in the inner layer at $t=6 \mathrm{~ns}$. $\mathrm{Al}$ and $\mathrm{Ni}$ are shown as blue (black) and yellow (white) spheres, respectively. The atomic arrangement is characteristic of a (110) orientation in NiAl.

stage II (from $t=3$ to $8 \mathrm{~ns}$ ), the number of bcc atoms abruptly increases together with temperature. The location of bcc atoms is in the inner layer. This behavior could be associated with an exothermic reaction between nickel and aluminum, giving rise to the formation of the compound $\mathrm{NiAl}$,

$$
\mathrm{Ni}+\mathrm{Al} \rightarrow \mathrm{NiAl} .
$$

If we make the strong hypothesis that the $\mathrm{Ni}$ atom keeps its cohesive energy until the reaction takes place, the energy balance expressed in terms of cohesive energies reads (see Table I)

$$
\frac{1}{2}\left[E_{0}(\mathrm{Ni})+E_{0}(\mathrm{Al})\right]=E_{0}(\mathrm{NiAl})+q,
$$

where $q=0.54 \mathrm{eV}$ is the excess of energy liberated by the atomic rearrangement. On the one hand, the number of $\mathrm{Al}$ and $\mathrm{Ni}$ atoms progressively reaches the 1:1 stoichiometry required to form the intermetallic [see Fig. 4(b)]. On the other hand, each atomic plane shows alternating lines of $\mathrm{Ni}$ and $\mathrm{Al}$ atoms in sectors of around $5-6 \mathrm{~nm}^{2}$ (see Fig. 5). This qualitative aspect is corroborated more quantitatively by the histogram of pairwise distances in each plane. In Fig. 6(a), the pairwise distances measured between $\mathrm{Ni}-\mathrm{Ni}$, $\mathrm{Al}-\mathrm{Al}$, and $\mathrm{Ni}-\mathrm{Al}$ bcc atoms are compared to the values expected for NiAl. We note an excellent agreement. It appears that the new atomic structure rests on a simple cubic lattice whose lattice parameter is very similar to the fcc lattice of Al. The distance $d_{i j}$ between $\mathrm{Al}$ planes in the middle layer remains close to the lattice parameter $a_{0}(\mathrm{Al}) / 2$. The fcc $\mathrm{Al}$ is characterized by 4 atoms/cell while the bcc NiAl cell contains only one atom of Al. Therefore, if we consider a build up of $\mathrm{NiAl}$ based on the existing $\mathrm{Al}$ lattice, only one $\mathrm{Al}$ atom from $\mathrm{fcc} \mathrm{Al}$ can stay in the new NiAl lattice. The maximum number of $\mathrm{Al}$ atoms in the middle layer that occupy bcc positions is thus limited to a quarter of the initial number (around 230 atoms in each plane). This is precisely what is observed in the simulation. Around half of the atoms (Ni or $\mathrm{Al})$ have a local lattice structure $a \neq 1$ and occupy the borders of the layers or 


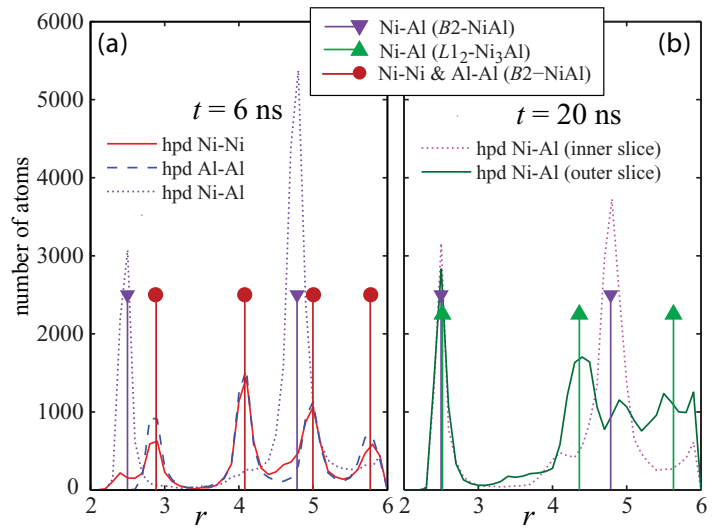

FIG. 6. (Color online) (a) Histograms of pairwise distances (hpd), at $t=6 \mathrm{~ns}$, between $\mathrm{Ni}$ and $\mathrm{Ni}$ (solid line), $\mathrm{Al}$ and $\mathrm{Al}$ (dashed line), and $\mathrm{Ni}$ and $\mathrm{Al}$ atoms (dotted line). (b) hpd, at $t=20 \mathrm{~ns}$, between $\mathrm{Ni}$ and $\mathrm{Al}$ atoms, in two representative slices of the inner (dotted line) and outer (solid line) layers. Vertical straight lines correspond to the theoretical values of the interatomic distances for $B 2-\mathrm{NiAl}$ and $\mathrm{L1}_{2}-\mathrm{Ni}_{3} \mathrm{Al}$. Distance is measured in angstroms.

the defect lines inside each slice. This corroborates the fact that only a local crystallization can spontaneously develop in the system. Around $t=4 \mathrm{~ns}$, the formation of the new phase $\mathrm{NiAl}$ has already taken place in atomic planes near the interfaces. At $t=6 \mathrm{~ns}$, all the planes show the crystallization phenomenon. As shown in Fig. 3, the system then reaches the maximum number of bcc atoms and stays on this plateau value for a while before a slow decrease in bcc atoms. The maximum temperature during stage II remains lower than $1100 \mathrm{~K}$.

Stage III, which covers the period from $t=8$ to $12 \mathrm{~ns}$, can be considered a transient stage. The rise in temperature becomes less steep $(10 \mathrm{~K} / \mathrm{ns})$ and the number of atoms in local bcc configuration decreases by $6 \%$ over this period. The measurement of the density along $z$ shows well distinct peaks [see Fig. 4(c)] even if locally some atoms may join the adjacent atomic plane in the outer layers (larger peaks). The $\mathrm{Al}$ atoms that diffuse in Ni occupy substitutional positions in the fcc lattice of nickel. Anyway, $\mathrm{Al}$ atoms with $a=2$ are observed close to the interfaces in the upper and lower layers. In these layers, the local stoichiometric ratio $x_{i}\left[x_{i}=n_{i}(\mathrm{Al}) / n_{i}(\mathrm{Ni})\right]$ is close to 0.33. A typical situation is illustrated in Fig. 7. One wonders if the solid solution will evolve and give rise to the formation of another phase. During the diffusion of $\mathrm{Al}$ atoms in the Ni substrate, $\mathrm{Al}$ atoms start to occupy vacancies liberated by diffusing $\mathrm{Ni}$ atoms. As soon as the number of $\mathrm{Al}$ atoms is locally sufficient ( $1 \mathrm{at} / \mathrm{cell})$, a local reorganization takes place with the formation of the $\mathrm{Ni}_{3} \mathrm{Al}$ compound. The balance energy for the cohesive energy per atom reads

$$
\frac{1}{4}\left[3 \times E_{0}(\mathrm{Ni})+E_{0}(\mathrm{Al})\right]=E_{0}\left(\mathrm{Ni}_{3} \mathrm{Al}\right)+q^{\prime}
$$

with $q^{\prime}=0.38 \mathrm{eV}$ the excess of energy liberated by the atomic rearrangement. Note that the exothermicity of this reaction is less than that for Eq. (1). This can partly explain the slower heating observed during stage III. Figure 8 shows the histogram of potential energy per atom for all atoms outside the inner layer. At the end of stage III $(t=12 \mathrm{~ns})$, the distribution becomes bimodal. The peak associated with

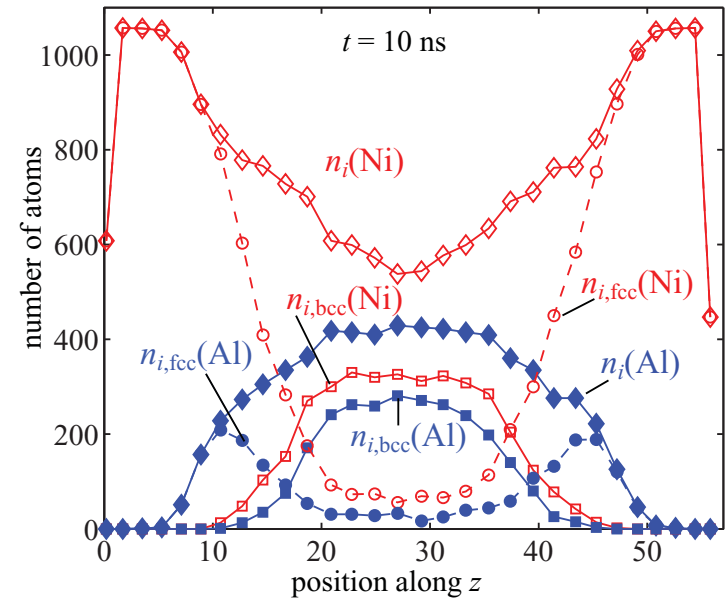

FIG. 7. (Color online) Local number of atoms $n_{i}$ in a slice $i$ along $z$ at $t=10 \mathrm{~ns}$. Ni species are represented by hollow markers and $\mathrm{Al}$ by full ones. The local number of atoms $n_{i}$ is indicated by diamonds, the local number of bcc atoms $n_{i, \text { bcc }}$ by squares, and the local number of fcc atoms $n_{i, \mathrm{fcc}}$ by bullets. Position is measured in angstroms.

pure Ni drops and a second peak appears around the value $E_{\mathrm{Ni}}$ in $\mathrm{Ni}_{3} \mathrm{Al}$. This demonstrates the formation of a new phase $\mathrm{Ni}_{3} \mathrm{Al}$ in the outer layers induced by the diffusion of $\mathrm{Al}$ in $\mathrm{Ni}$ substrate.

Due to the specific geometry of our model, the system is subjected to a wetting phenomenon. During stage I, Al atoms wet the free surfaces of nickel. The inner layer of aluminum slips and spreads on the nonrepellent free Ni surfaces at the borders of the system. This allows the $\mathrm{Al}$ layer to freely adjust the misfit of the lattice parameter. The wetting induces the defects, observed from the first moments of the simulation, which will play an essential role in the progress of diffusion and, actually, of reaction. By the way, the outer Ni layers, submitted to periodic boundary conditions, may be considered as a rigid substrate. The importance of wetting is measured by the number of atoms $n_{w}$ outside a central parallelepiped

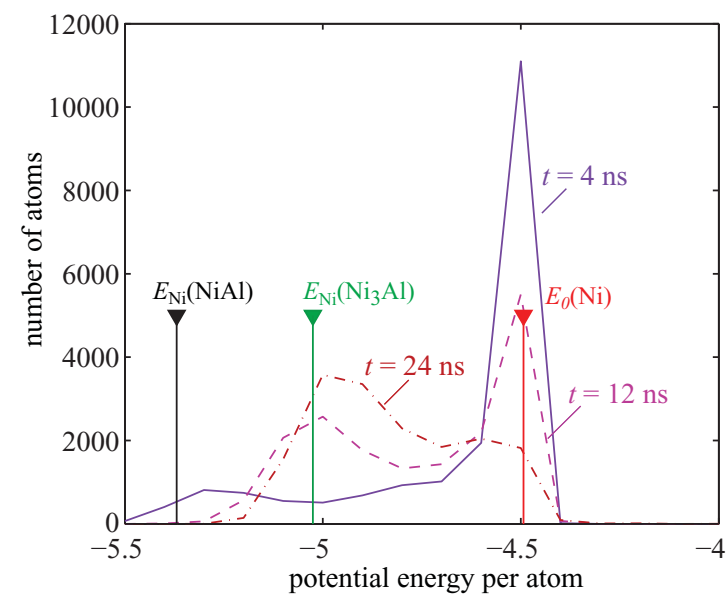

FIG. 8. (Color online) Histogram of potential energies per atom for all the Ni atoms in the outer layers. Full line, dashed line, and dashed-dotted line correspond to $t=4,12$, and $24 \mathrm{~ns}$, respectively. Vertical straight lines indicate the theoretical values of the potential energy per atom in a given phase. Energy is measured in $\mathrm{eV}$. 
([0,90], [0,90],[18.6,37.6]) (see Fig. 3). As soon as Ni starts to diffuse in the Al layer, $\mathrm{Ni}$ atoms are also found in the wetting zone. During stage II, $n_{w}$ is decreasing. This is due to the formation of $\mathrm{NiAl}$, whose density is larger than pure Al. The wetting phenomenon is stable during stage III. Contrariwise, $n_{w}$ sharply increases after $t=12 \mathrm{~ns}$. Most of them are $\mathrm{Ni}$ atoms. We will see that this evolution stage corresponds to the formation of $\mathrm{Ni}_{3} \mathrm{Al}$ in the outer layers. Since the density of $\mathrm{Ni}_{3} \mathrm{Al}$ is much lower than the density of pure Ni (see Table I), the ejected $\mathrm{Ni}$ atoms will occupy the free space left around the inner layer.

The long-time behavior is associated with stage IV, from $t=12$ to 24 ns. A slow heating is still observed $(5 \mathrm{~K} / \mathrm{ns})$ while the number of atoms in a local bcc configuration is decreasing and the number of atoms in an fcc local environment is relatively stable. The careful study of the atomic planes gives a good insight into the system's evolution. Well-defined density peaks demonstrate the persistence of a solid-state evolution. Even if the system is heated during the progress of the reaction, the temperature stays lower that the melting temperature of $\mathrm{Ni}, \mathrm{NiAl}$, and $\mathrm{Ni}_{3} \mathrm{Al}$ even at such nanoscales. Aluminum is supposed to melt around $1000 \mathrm{~K}$ for nanosystems. Note that, in the nanolayered system considered, $\mathrm{Al}$ has already reacted with $\mathrm{Ni}$ before the temperature has reached this value. This prevents the system from melting. In the inner layer, bcc atoms progressively disappear close to the interface while bcc atoms are still present in the core. In the outer layers, most of the $\mathrm{Al}$ atoms that have migrated have an fcc local configuration. They are first located near the interfaces, then increasingly far. Moreover, for these slices, the stoichiometric ratio $x_{i}$ is close to the one expected for $\mathrm{Ni}_{3} \mathrm{Al}$. The histogram of the potential energies per atom computed for $\mathrm{Ni}$ atoms outside the central box shows that the peak centered around $E_{\mathrm{Ni}}\left(\mathrm{Ni}_{3} \mathrm{Al}\right)$ becomes dominant. Moreover the hpd, calculated in a representative slice in the outer layer, reveals that the $\mathrm{Ni}-\mathrm{Al}$ interdistance is located around the one expected for $\mathrm{Ni}_{3} \mathrm{Al}$ [see Fig. 6(b)]. Contrariwise, the same analysis inside the inner layer shows the persistence of $\mathrm{NiAl}$. It is interesting to follow how $\mathrm{Al}$ atoms will invade the overall system. The thickness of the layer containing $\mathrm{Al}$ atoms increases by successive jumps. This shows the progressive diffusion of $\mathrm{Al}$ in the Ni substrate and, by the way, the possibility of phase transformation in $\mathrm{Ni}_{3} \mathrm{Al}$.

\section{CONCLUSION}

In this system, the crystallization of $\mathrm{NiAl}$ is spontaneous since it develops even in the absence of any nucleus. The $\mathrm{NiAl}$ compound first appears at the Ni-Al interface. But the existence of line defects in atomic planes promotes the rapid diffusion of $\mathrm{Ni}$, which is further enhanced by the rise in temperature associated with the formation of $\mathrm{NiAl}$. The $\mathrm{NiAl}$ phase formed in the core of the inner layer self-organizes in the small area $\left(6 \mathrm{~nm}^{2}\right)$ delimitated by the line defects. Atoms of aluminum leaving the inner layer react with the nickel of the outer layers to form a new phase rich in nickel, $\mathrm{Ni}_{3} \mathrm{Al}$, near the interfaces. The new layer develops in the direction perpendicular to the interface. The phase growth is limited by the diffusion of Al thorough the new phase and takes place layer by layer. Concomitantly, $\mathrm{Ni}$ atoms diffuse in this opposite direction. The excess of $\mathrm{Ni}$ in the substrate acts as a reservoir, which maintains the system out of equilibrium. As a consequence, the diffusion flux of $\mathrm{Ni}$ in the inner layer partly destabilizes the $\mathrm{NiAl}$ formed at the very beginning of the reaction.

Our study enables us to detect the mechanisms responsible for the reaction in thin films at nanoscales and to analyze the microstructure obtained after the reaction. This approach could be valuably extended to understand the influence of the films' thickness, stoichiometry between metals, or initial temperature.

\section{ACKNOWLEDGMENTS}

The authors would like to thank S. Garruchet and A. L. Garcia for helpful discussions. The use of computational facilities at the Computing Center of the University of Bourgogne, DSI-CCUB, is gratefully acknowledged.
${ }^{1}$ T. P. Weihs, in Handbook of Thin Film Process Technology, edited by D. A. Glocker and S. I. Shah (IOP, Bristol, 1998).

${ }^{2}$ J. Wang, E. Besnoin, A. Duckham, S. J. Spey, M. E. Reiss, O. M. Knio, and T. P. Weihs, J. Appl. Phys. 95, 248 (2004).

${ }^{3}$ A. J. Swiston Jr., E. Besnoin, A. Duckham, O. M. Knio, T. P. Weihs, and T. C. Hufnagel, Acta Mater. 53, 3713 (2005).

${ }^{4}$ A. S. Rogachev, Russ. Chem. Rev. 77, 21 (2008).

${ }^{5}$ P. Geysermans, D. Gorse, and V. Pontikis, J. Chem. Phys. 113, 6382 (2000).

${ }^{6}$ F. Delogu, Nanotechnology 18, 065708 (2007).

${ }^{7}$ A. Kerrache, J. Horbach, and K. Binder, Europhys. Lett. 81, 58001 (2008).

${ }^{8}$ B. J. Henz, T. Hawa, and M. Zachariah, J. Appl. Phys. 105, 124310 (2009).

${ }^{9}$ S. Zhao, T. C. Germann, and A. Strachan, J. Chem. Phys. 125, 164707 (2006).
${ }^{10}$ S. Zhao, T. C. Germann, and A. Strachan, Phys. Rev. B 76, 104105 (2007).

${ }^{11}$ N. S. Weingarten, W. D. Mattson, and B. M. Rice, J. Appl. Phys. 106, 063524 (2009).

${ }^{12}$ S.-G. Lee and Y.-C. Chung, J. Appl. Phys. 105, 034902 (2009).

${ }^{13}$ X. W. Zhou, H. N. G. Wadley, R. A. Johnson, D. J. Larson, N. Tabat, A. Cerezo, A. K. Petford-Long, G. D. W. Smith, P. H. Clifton, R. L. Martens, and T. F. Kelly, Acta Mater. 49, 4005 (2001).

${ }^{14}$ R. A. Johnson, Phys. Rev. B 39, 12554 (1989).

${ }^{15}$ Y. Mishin, M. J. Mehl, and D. A. Papaconstantopoulos, Phys. Rev. B 65, 224114 (2002).

${ }^{16}$ See [http://lammps.sandia.gov/]; S. Plimpton, J. Comput. Phys. 117, 1 (1995).

${ }^{17}$ G. J. Ackland and A. P. Jones, Phys. Rev. B 73, 054104 (2006). 